\title{
DESIGN OF SPECIMENS FOR SHORT-TIME “FATIGUE" TESTS
}

\author{
By L. B. Tuckerman and C. S. Aitchison
}

\section{ABSTRACT}

This paper discusses the controlling factors in the design of short-time "fatigue" test specimens, which differ from those of the endurance-run type of fatigue test. In endurance runs it is necessary to secure failure at a place where the stresses are determinate and calculable. In short-time "fatigue" tests failure is not desired.

It is possible, then, to design the short-time "fatigue specimen with maximum stresses uniform over a large portion of the material, thus securing greater sensibility. This should be the controlling factor in the design of these specimens.

Specimen shapes are shown suitable for different types of short-time "fatigue" tests. Because of the simple specimen shape the Sondericker type of machine is considered best suited for these tests.

\section{CONTENTS}

I. Introduction

II. Principles of specimen design in endurance-run "fatigue" testing - 48

III. Principles of specimen design in short-time "fatigue" testing....... 50

IV. Design of short-time "fatigue" test specimens for different types of fatigue machines.-..... 51

V. Conclusions_._. 55

\section{INTRODUCTION}

There are two radically different ways in which fatigue tests are made. The first is the classical endurance test of Wöhler, ${ }^{1}$ a fatigue test proper, in which a specimen is subjected to repeated variations of stress until failure. The second is the short-time fatigue test, either by theload-deflection method of Smith or the rise-of-temperature method of Stromeyer. The work of J. H. Smith, ${ }^{2}$ H. J. Gough, ${ }^{3}$ F. C. Lea, ${ }^{4}$ and others with the load-deflection method, and C. E. Stromeyer, ${ }^{5}$ H. J. Gough, ${ }^{3}$ H. F. Moore, ${ }^{6}$ and others with the temperature-rise method indicates that for some ferrous materials, at least, these short-time tests give results consistent with those obtained from endurance runs.

\footnotetext{
1 Wŏhler, A., Zeitschrift für Bauwesen, 8, p. 642; 1858; 10, p. 583, 1860; also, Engineering, London, 11; 1871.

' Smith, J. H., The Journal of the Iron and Steel Institute, No. 2, 1910, p. 246.

${ }^{3}$ Gough, H. J., Tho Engineer, London, Aug. 12, 1921.

4 Lea, F. C., Engineering, London, Feb. 16, 1923, p. 217; Feb. 23, 1923, p. 252.

$\checkmark$ Stromeyer, C. E., Royal Society Proceedings, 1914.

- Moore, H. F., An investigation of the fatigue of metals, Univ. of Illinois Bulletin No. 12t; also series of $1922,136$.
} 
Some experiments on nonferrous materials and high tensile strength steels make it seem doubtful if any general relation exists between the results of these short-time tests and endurance runs. The question, however, deserves further experimental investigation.

The shape of the specimens in these tests determines in a large measure their reliability, and in the case of the short-time test, also, their sensibility. Examination of published papers shows that, while the shape of specimen in fatigue tests by endurance runs has received careful consideration, apparently less attention has been paid to the shape of the specimen used in the short-time tests. A similar shape has generally been used for these latter, although the considerations which should determine it are entirely different in the two types of test. Heretofore very sensitive optical lever systems have been used to observe the break in the load-deflection curve. By suitably designing the specimens it has been found possible to detect this break readily with an ordinary dial micrometer:

\section{PRINCIPLES OF SPECIMEN DESIGN IN ENDURANCE-RUN "FATIGUE" TESTING}

In endurance runs, for all types of machines, whether rotating beam, alternating flexure, pure tension compression, or alternating torsion, the specimen is designed to insure breaking at a point where the stress is calculable. Near the clamps by which the specimen is fastened and through which the load is applied, there are unavoidably introduced local indeterminate stresses which are, in general, greater than those in neighboring parts of the specimen.

If, in addition, the cross section of the specimen changes rapidly, as at a fillet, the stresses though determinate are not calculable by any available methods.

The shape of the specimen must provide for a maximum of stress sufficiently removed from clamps and fillets to be calculated by the elementary Euler-Bernoulli theory and large enough to exceed any local stresses near the clamps and fillets existing under the standard clamping procedure. This is accomplished by varying the crosssectional area gradually along the specimen. Figure 1 shows five specimens used for endurance runs. The positions of the clamps are shown by the cross-hatched areas. The dotted line above the specimen shows in each case the approximate stress distribution along the specimen.

The highest maximum of stress lies on a free portion of the specimen far enough from the clamps to be calculated with sufficient accuracy by elementary theory. This maximum has been adjusted empirically by shaping the specimen until failure takes place in the free span and not in the region of indeterminate stress near the clamps. 

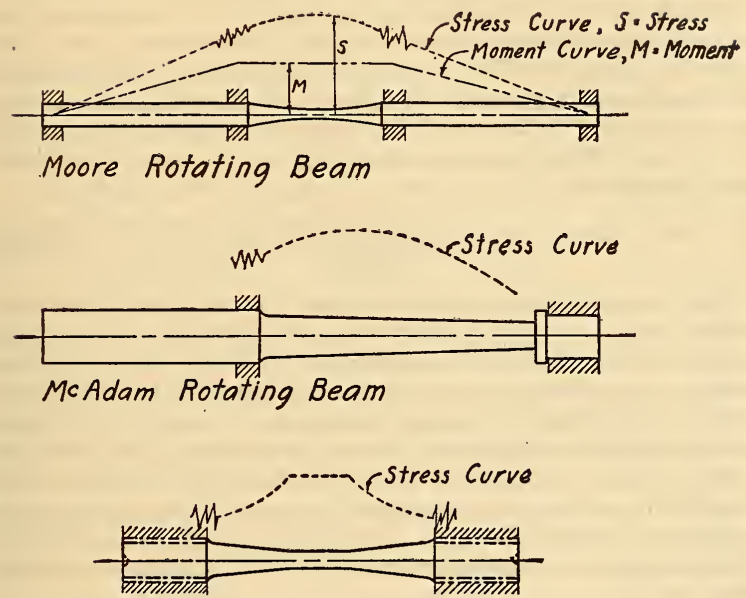

Haigh Tension Compression

Gough Torsion

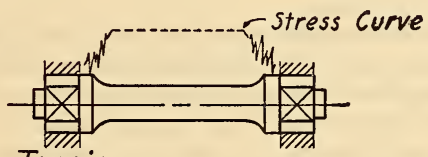

\section{Smith Tension Compression}

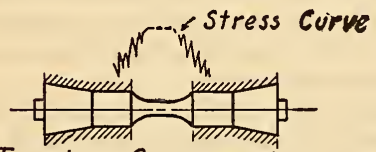

Fra. 1.-Specimen shapes for endurance-run fatigue tests 
The necessity for having the stress calculable and also a maximum in a portion of the test specimen, so that failure will occur in that portion, has led to these designs in which the maximum stress is localized over a small portion of the length of the specimen. For example, in the Moore specimen for the Sondericker ${ }^{7}$ type of machine, the stress falls off 1 per cent within one-tenth of an inch from the location of maximum stress, while in the McAdam ${ }^{8}$ specimen for the Wöhler ${ }^{3}$ type of machine $11 / 2$ per cent within three-quarters of an inch. Definite values are not given by other investigators, but the specimen shapes shown have similar characteristics.

\section{PRINCIPLES OF SPECIMEN DESIGN IN SHORT-TIME "FATIGUE" TESTING}

The specimen shape for the short-time "fatigue" test in the rotating beam "fatigue" machine, either by the temperature rise or the loaddeflection method should, in general, be of different design from that used in the endurance run. In this short-time test the "fatigue" limit is determined by applying increments of load to the rotating specimen and recording either the temperature rise or the deflection. The addition of each increment of load during the first part of the test will cause a nearly proportional increase in temperature rise or deflection. Finally, however, a load will be reached at which the temperature rise or deflection readings increase much more rapidly than the load.

The stress calculated at this load is, for some ferrous materials at least, consistent with the fatigue limit as obtained from endurance runs on identical material.

These load temperature rise or load deflection readings show typical curves, such as $A$ and $B$, Figure 2, which were plotted from loaddeflection tests on two differently shaped specimens of nickel steel. The ordinates for these curves are the loads applied and the abscissas may be either temperature or deflection increments. These two curves show that at equal increments of load the increments of deflection or temperature rise are equal and remain so until some load is reached at which the increments of deflection or temperature increase.

The factors of specimen design which result in curves either of the type $A$ or $B$ will now be discussed.

If all of the material of the specimen were uniformly stressed by the machine, the curve obtained would resemble $B$, since all would reach the critical stress at the same load, and consequently the break in the curve would be sharp and definite. When, however, the specimen is designed as for an endurance-run test where a failure is ex-

7 Sondericker, J., "Description of some repeated stress experiments," Tech. Quart., April, 1892.

${ }^{8}$ McAdam, D. J., jr., "Endurance of steel under repeated stresses," Chem. and Metl. Eng., Dec. 14, 1921.

9 See footnote 1, p. 47 . 
pected, provision is made to localize the maximum stress over a small portion of the surface. Then, as the load is increased, at first only a relatively small portion of the material reaches the critical stress, while all the rest is stressed below that limit. As the loading proceeds this small portion of the material is overstressed before the adjacent material reaches the critical stress. The action is progressive and cumulative until an increment of temperature or deflection is obtained sufficiently greater than those preceding to be detected on the curve. The curve obtained will resemble $A$ of Figure 2 .

It is difficult on a curve such as $A$ to determine closely the stress at which the deviations from a constant value pass the limit of the unavoidable irregularities due to experimental error.

The obvious advantage of the sharp and definite break of $B$ over the gradual curvature of $A$ points to the desirability of so designing specimens for short-time "fatigue" tests that as large a portion of the material as practical shall be uniformly stressed. In these shorttime tests the specimen is not broken, and unless the time of application of each increment of load is so great that the tests take on somewhat the character of endurance runs there is, therefore, no necessity of providing a maximum of stress on a free portion of the surface. In addition, the indeterminate stresses concentrated in the neighborhood of the clamps contribute only a small part to the deflection or temperature rise so that there is no practical disadvantage in allowing them to be higher than those in the uniformly stressed free portion of the specimen. Those limitations which determined the design of endurance-run specimens, therefore, do not apply to the short-time tests. Consequently, it is possible so to shape the specimen that the stress is uniform over a much larger portion and the curves will more nearly resemble $B$ than $A$, thus giving greater sensibility. This uniformity of stress should, therefore, be the controlling factor in the design of short-time "fatigue" test specimens.

\section{DESIGN OF SHORT-TIME "FATIGUE" TEST SPECIMENS FOR DIFFERENT TYPES OF FATIGUE MACHINES}

In the Sondericker type of fatigue machine the specimen is a simple beam with two symmetrical concentrated loads. The moment curve for a machine loaded under these conditions is shown in Figure 1. The moment increases from each of the end reactions to the points of load and is constant between the loads. The greatest sensitivity and sharpest break in the load-deflection curvo will obviously be obtained by a thin-walled tube, such as shown in Figure $3, A$, whose shape, from the reaction points to the load points, is given by the equation

$$
x=k \frac{d^{4}-d^{4}}{d}
$$




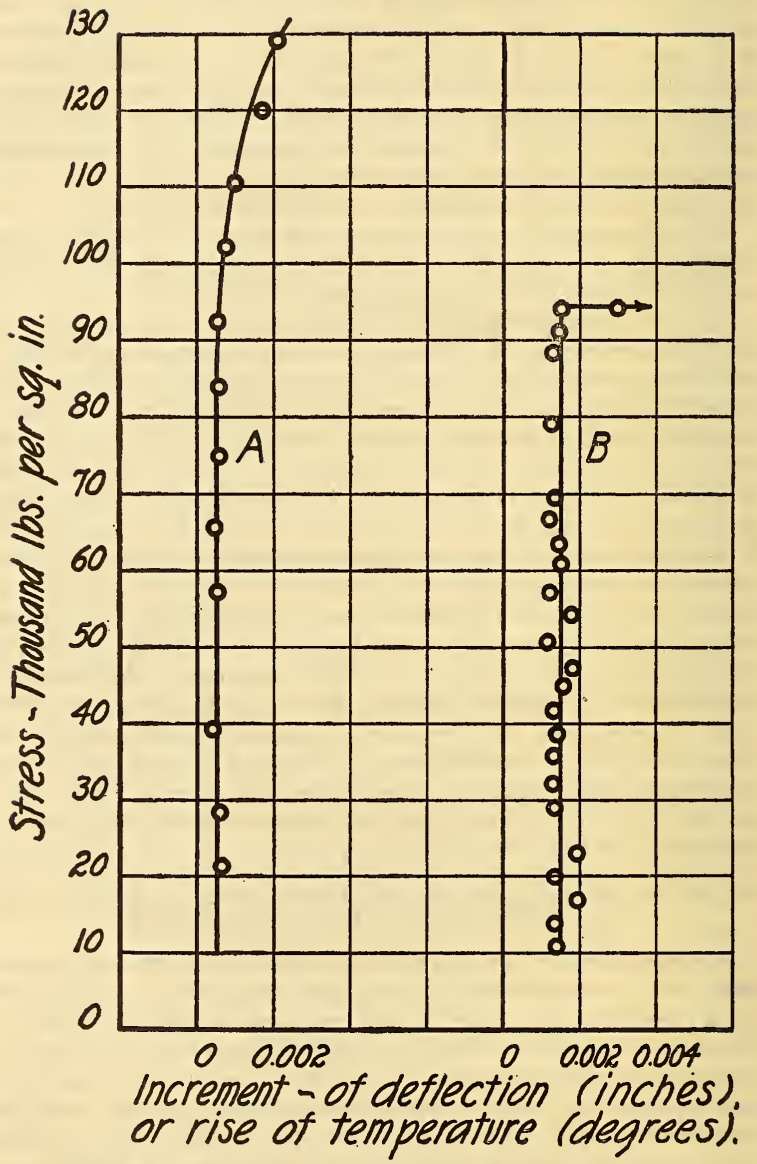

FIG. 2.-Stress increment of deflection or rise of temperate curves 

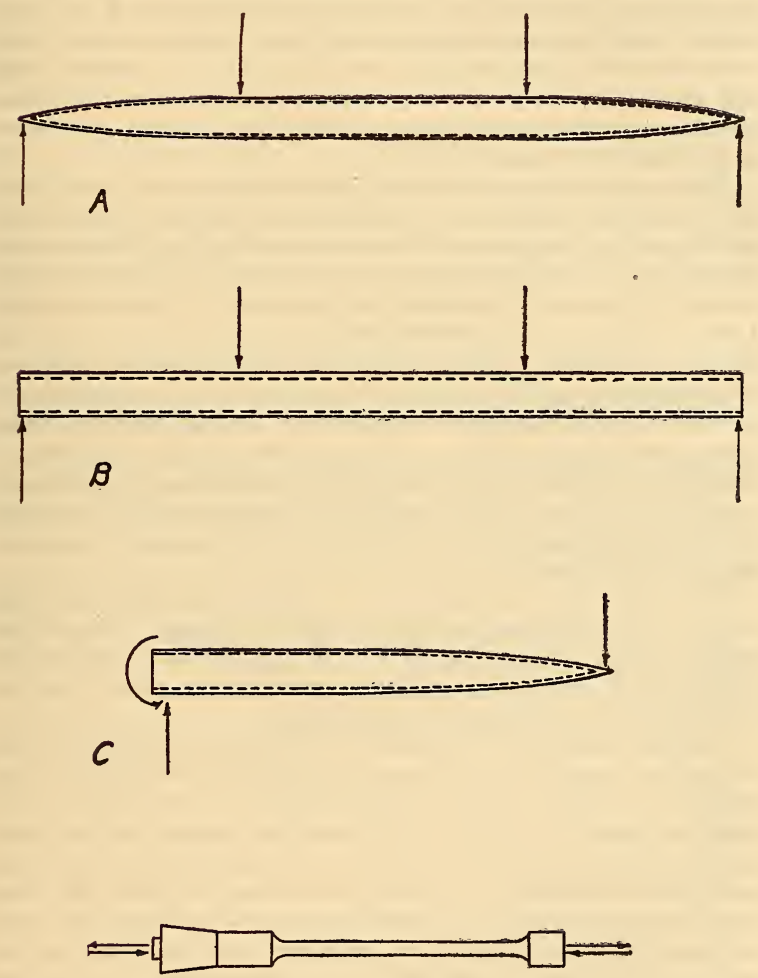

D

FIG. 3.-Specimens for short-time "fatigue" tests 
where $d$ is the outside diameter, $d_{1}$ the inner diameter, $x$ the distance from support, and $k$ a constant. Between the loading bearings the specimen will be simply a hollow cylinder. It is quite obvious that this shape is impracticable. Another somewhat simpler type that suggests itself is the hollow cylinder shown in Figure 3, $B$, but here, again, the difficulty encountered in uniformly boring out a long specimen of small outside diameter renders it expensive for routine testing and, in addition, the thin wall is easily deformed or otherwise abused and, if not perfectly symmetrical and true when tested, causes excessive vibrations in the machine.

The practical shape of specimen which suggests itself is the simple solid cylindrical specimen. This has the advantages of ease of machining, with a large amount of material reaching the critical stress at the same load. Specimens of ferrous materials of this shape have been tested and the results plotted as load increment of deflection curves. Curve $B$, Figure 2, was plotted from data obtained in a test of this kind on nickel steel. These results were obtained with an ordinary dial micrometer, graduated to 0.001 inch, reading the deflection of one of the loading bearings, instead of with the very sensitive optical lever systems used by others. The fact that so sharp and definite a break can be obtained with a relatively insensitive measuring system shows that the extra cost of machining the theoretically more desirable specimen shapes would not be justified by the added sensitivity obtained from them.

The sensitivity is not appreciably lowered by having the cylindrical portion between load points slightly reduced. The reduced section, however, should be as long as practicable. This has the advantage of preventing occasional breaks at the clamp when repeated tests are made on the same specimen.

The same considerations applied to the Wöhler, or cantilever, type of machine require that the specimen (fig. $3, C$ ) should be a thinwalled tube, whose shape can be expressed by the formula previously mentioned. It will be noted from this formula that the outer surface of the specimen may vary in diameter while the inner surface remains constant, or the inner surface may vary while the outer surface remains constant, or both inner and outer surface may vary in diameter. The difficulty of machining out the inner portion renders this shape impracticable for routine testing. It would be possible, however, to machine a solid specimen having the outer surface in the shape of a cubic paraboloid which seems to be the type of specimen best suited for this machine. McAdam's conical specimen will not be satisfactory because the maximum stress occurs on a relatively small portion of the surface of the material. The cylindrical specimen would be even less satisfactory because the maximum stress occurs near the reaction bearing. 
Since it is possible to obtain adequate sensibility with the simplest type of specimen, the Sondericker machine seems better suited to "short-time fatigue" tests than the cantilever machine.

In alternating torsion tests the hollow cylinder obviously would give the greatest sensibility and probably the solid cylinder would give adequate sensibility. Specimens approximating these were used by Gough.

For tension-compression tests any prismatic shape gives uniform stress throughout the specimen. The solid cylinder is as advantageous as any other form. The shape of specimen, shown in Figure $3, D$, was one used by J. H. Smith in obtaining stress-strain measurements with a tension compression type of fatigue machine. Smith does not fully discuss his reasons for making the specimen of this shape, but it is evident that the requirements for sensitivity are fulfilled.

\section{CONCLUSIONS}

1. The need of short-time tests to determine the fatigue characteristic of metals makes it desirable to investigate the so-called shorttime "fatigue" tests.

2. Among these short-time tests the load deflection and load temperature rise methods of test seem to promise to be of value for limited classes of material and, therefore, deserve further study.

3. With suitably designed specimens a higher sensibility can be obtained in these short-time "fatigue" tests enabling simpler measuring instruments to be used.

4. The Sondericker type of rotating beam fatigue machine with a plain cylindrical specimen or a specimen with a slightly reduced cylindrical section between load bearings seems best suited for these tests, combining high sensibility with a simple specimen shape.

Washington, June 5, 1924. 